\section{Clinical Analyses}

Wild and Cultivated Plants: A Natural Source of Psychoactive Substances Used by Young People-Clinical Reports from Poison Control Centre of Milan

P. A. Moro ${ }^{1}$, R. Borghini ${ }^{1}$, V. Di Masi ${ }^{1}$, T. Giarratana ${ }^{1}$, M. Bissoli ${ }^{1}$, A. Travaglia ${ }^{1}$ and F. Cassetti ${ }^{2}$

${ }^{1}$ Poison Control Centre, Niguarda Ca' Granda Hospital and ${ }^{2}$ Herbal Techniques, Faculty of Pharmacy, University of Milan, Italy

Several psychoactive species have been used for centuries as a part of religious rituals or as medicines. These drugs are now being used recreationally by young people both because plants are considered safer than chemicals and because most of them are not illegal to use, possess, grow or distribute and cannot be detected by specific laboratory drug tests. Despite being 'natural substances' they can produce severe toxic effects, such as coma, seizures or cardiac dysrhythmias. We reviewed all the calls concerning suspected plant poisonings collected from poison control centre (PCC) of Milan between 2001 and 2005 to point out the cases related to recreational use of psychoactive species. Hallucinogen mushrooms and Cannabis spp. were not included in this search. Among 4580 calls concerning plant poisonings, 41 were closely connected to the recreational use of psychoactive species. The age range was 16-36. Datura stramonium, Argyreia nervosa, Myristica fragrans, Salvia divinorum, Mandragora officinarum were the species involved. The recreational use of psychoactive plants among young people in Italy is difficult to assess because of the lack of epidemiological data. The assumption of such natural drugs would be suspected in young people showing acute psychiatric, neurological or cardiac symptoms, especially when urinary drug test results negative. The identification of clinical cases by PCCs and their report to Health Authorities is of primary importance for making preventive public health interventions, such as the recent insertion of $A$. nervosa and $S$. divinorum in the list of psychotropic substances under Law control.

Electroacupuncture Therapy in the Climateric Syndrome Treatment: A Randomized Controlled Trial-Preliminary Results

S. Baccetti ${ }^{1}$, E. Buiatti ${ }^{2}$, M. Da Frè ${ }^{2}$, M. Faedda ${ }^{1}$, A. Guerrera ${ }^{1}$ and R. Munizzi ${ }^{1}$ ${ }^{1}$ Referring Center for Complementary Medicine, Tuscany Region, V.. Pistoiese 185, San Donnino, Campi Bisenzio, 50017 and ${ }^{2}$ Unit of Epidemiology, Regional Health Agency of Tuscany, Florence, V. Vittorio Emanuele II 64, 50134, Italy

Menopause is a physiological phase of women's lifetime. However, a certain number of women suffer from symptoms such as hot flashes, vaginal dryness and mood swings. Physicians today have a much more prudent approach towards HRT (hormone replacement therapy) and women are more and more frequently choosing treatments with scarce or no side-effects. Acupuncture is widely used in many countries for the treatment of these symptoms. This study, a controlled randomized clinical trial with a follow-up at 4 months after the end of treatment, wants to contribute to define acupuncture's efficacy in the treatment of menopausal symptoms, since so far we have had an insufficient number of clinical trials about this topic. A total of 100 women recruited (age range 45-56 years, physiological menopause, hot flashes at least 3-4 times daily, not treated with HRT the last month) have been divided in two randomized groups. The first group (group A) was treated with TCM (traditional Chinese medicine) after the recruitment. In this group, the AOGOI questionnaire was completed at recruitment and at the end of the treatment by a personal interview. The second group (group B) is the control group, and was treated 30 days or more after the recruitment. During these 30 days or more women of group $B$ did not receive any treatment nor placebo. In group B, the AOGOI questionnaire was completed at recruitment and after 30 days or more of no treatment and at the end of the treatment by a personal interview. The treatment in the two groups was the same. Preliminary results regarding 59 women ( 38 in the A group and 21 in the control group) are as follows: after the treatment the group A was significantly better than the control group (group B considered after 30 days or more or no treatment) about the improvement of the health, both general $(P<0.001)$ and related to the menopausal symptoms $(P<0.001)$.
Results of the Research with an Extract of Mangifera Indica L. (Vimang): from Ethnomedicine to Clinical Trials M. Guevara, A. Álvarez, A. Riaño, G. Pardo, G. Reynaldo, G. Garrido and R. Delgado

Biomedical Research Department. Centre of Pharmaceutical Chemistry. 200 street and 21 avenue, Atabey, Postal Code 11600. Havana City, Cuba

For more than a 100 year the plants have been used for medicinal purposes. Mango (Mangifera indica L.), which belongs to the Anacardiaceae family, is widely found in Cuba and in many other tropical and sub-tropical regions. Mango stem bark has been traditionally used like aqueous extract, obtained by decoction, in ethnomedical practice. The Centre of Pharmaceutical Chemistry, from Cuba, has within its high-priority lines of work the introduction of this natural product for medical use. The investigations with this natural extract of the bark of the mango tree $(M$. indica $L$.) have been focused on obtaining the standardized extract, the pharmacological and toxicological studies, the industrial scale-up of the active component and the studies of pharmaceutical technology (Vimang). All clinical trials and ethnomedical studies with Vimang were retrieved. The first clinical investigation was made with the objective to know the traditional applications, the effectiveness and the potentialities of the extract of $M$. indica $L$. in our conditions. After that, our institution began the development of a strategy of clinical evaluation, for demonstrating the therapeutic effectiveness of the extract in diverse pharmaceutical forms through controlled clinical trials in diseases with an important inflammatory or/and oxidative stress component that guarantee the introduction of Vimang like phytomedicine. The results obtained from the investigations carried out with Vimang have been the following: (i) manifestation of an antioxidant activity of the extract, similar or superior to vitamins C, E and other natural products; (ii) the evidence of the hepatoprotective effect in proteins and hepatic microsome, as well as in hepatic ischemia-reperfusion in rats; (iii) the demonstration of the neuroprotector effect in neuronal cells of hippocampal CA-1 region in assay of cerebral ischemia-reperfusion in Gerbils. (iv) the demonstration of the anti-inflammatory mechanism of action through the inhibition of prostaglandins, leukotrienes, phosfolipase $\mathrm{A}_{2}$, tumor necrosis factor and radical nitric oxide. ( $v$ ) the national readiness of the vegetable raw material, with a minimum environmental impact, with a stable and standardized chemical composition. (vi) the non-toxicity of the product for oral and topical pathway, demonstrated in acute and sub-chronic studies, as well as the non-irritability for oral, topical, ophthalmic, rectal and vaginal pathways, and the absence of mutagenic and clastogenic effects. Good results were found in patients with AIDS, in old person, in skin illnesses and blood coagulation.

The Relevance of Protein Substitution During Abrosia: A Pilot Study Peter W. Gündling

Europa Fachhochschule Fresenius, University of Applied Sciences, Limburger Str. 2, D-65510 Idstein

In complementary medicine, proteins are regarded with contradictory significance. On the one hand they are the most important constituents of the human organism, and essential amino acids in particular do have multiple health protecting and health promoting properties. On the other hand protein deposits are regarded as the cause for several chronic diseases. The lower limit of daily protein ingestion is $0.6 \mathrm{~g}$ protein $/ \mathrm{kg}$ body weight. If the ingestion of protein falls below this limit-as during fasting cures-muscular atrophy will be the result. During the last 2 years 120 patients who performed a fasting cure according to F.X. Mayr were subject to analysis of body tissue using a near-infrared (NIR) measuring method both before and after a 3-week period of fasting cure. Moreover, subjects underwent several clinical and laboratory tests and were assessed for wellbeing. Patients were assigned to two groups. One group received the standard milk and bun diet and the other group received the standard diet with additional $10-20 \mathrm{~g}$ of whey protein daily. The group with standard diet only showed a mean loss of muscle tissue of $800 \mathrm{~g}$ while the whey protein group showed a mean loss of muscle tissue of $200 \mathrm{~g}$. There was no significant difference in any of the other clinical parameters that were tested. Subjective statements of wellbeing were more positive in the whey protein group during the fasting period. Results of the study indicate that patients benefit in two ways during abrosia from additional protein supply: The treatment with protein of high biological valence protects the patients from muscular atrophy and increases the wellbeing during the cure. 


\section{Six Cases of Severe Adverse Reactions to Cervical Chiropractic} Manipulations

L. Gori ${ }^{1}$, L. Guidi ${ }^{2}$, L. Bartolozzi $i^{2}$, S. Muugnai ${ }^{2}$, M. Lombardi ${ }^{2}$, S. Brotini ${ }^{2}$ and F. Firenzuoli ${ }^{1}$

${ }^{1}$ Center of Natural Medicine, Service of Chirotherapy and ${ }^{2} \mathrm{OU}$ Neurology

S. Giuseppe Hospital, Empoli, Italy

The growing recognition of cervical manipulation as a treatment of neck pain and cervicogenic headaches has lead to increased interest in potential complications that may result from this treatment approach. Minor side-effects associated with chiropractic are common. Recent surveys have reported that many neurologists encounter cases of severe adverse reactions that occur at various times after cervical manipulation, whereas most practitioners of spinal manipulation are of the opinion that these events are extremely rare. We report six cases of severe adverse reactions following chiropractic manipulation: (i) Female, 35-year old. Suffering of cervical pain underwent chiropractic cervical manipulation. Sudden permanent torticollis. The patient referred that the chiropractic did not see her radiography of the neck and that she underwent other 30 treatments under the same health-caregiver without any improvement Follow-up >2 years: she is still suffering of chronic unremitting torticollis. (ii) Male, 63-year old. Suffering of cervical pain after chiropractic manipulation reported dissection of the left vertebral artery. Follow-up $>1$ year: dysarthria and ataxia. (iii) Female 28-year old. Underwent chiropractic cervical manipulations for cervical pain and headache. She reported expulsion of the disk in $\mathrm{C} 6-\mathrm{C} 7$ and myelopathy. Follow-up $>1$ year: pyramidal signs in lower limbs and tactile hypoestesia. (iv) Female 68-year old. The patient was suffering of cervical pain and headache and had a previous diagnosis of breast cancer. After chiropractic manipulation fracture of a vertebra. Follow-up: quadriplegia and death. (v) Male 53-year old. Underwent chiropractic manipulation for cervical pain, reported right internal carotid dissection. Follow-up $>1$ year: the patient is fully recovered. (vi) Female 66-year old. The patient had unremitting cervical pain. After chiropractic manipulation reported right internal carotid dissection. Follow-up $>2$ years: the patient fully recovered. Chiropractic manipulation should be executed only after a detailed anamnesis and neurological examination, to avoid not only severe adverse reactions that probably are only the tip of the iceberg, but also minor complaints and other not severe adverse reactions that should not be considered but only negligible effects of chiropractic manipulations.

\section{An Overview on Echinophora platyloba as a Weekly Antimicrobial but Potent Antifungal Agent \\ Majid Avijgan ${ }^{1}$, M. Mahbobi ${ }^{2}$, M. Darabi ${ }^{3}$, M. Saadat ${ }^{1}$, M. Hafizi ${ }^{1}$ and}

M. A. Nilforosh Zadeh ${ }^{3}$

${ }^{1}$ Shahr-e-Kord University of Medical Sciences, Shahr-e-Kord, ${ }^{2}$ Barij Essence

Pharmacology Company, Isfahan and ${ }^{3}$ Tehran University of Medical Sciences, Tehran, Iran

There are many Iranian plants used in folk medicine, one of them being Echinophora platyloba $D C$. This review presents the results of our five previous studies showing the effectiveness of $E$. platyloba extract's on some fungal and bacterial agents. The plant was collected. The micro-organisms used in these studies were purchased from Biotechnology Research Center of Iran, Tehran. After obtaining ethanolic extract (5\%) and by using micro broth dilution method, the disc containing $20 \mu \mathrm{g}$ of extract was compared with standard disc of antibiotic (Himedia Company) of Kefline, Penicillin G and Cloxaciline against some Gram-positive bacteria. In other study, with having five tubes of differen diluted solution of hydro alcoholic extract as: $0,35,50,150,250 \mathrm{mg} / \mathrm{ml}$ and by using agar dilution method, the yeast of Candida albicans and fung (Dermatophyte) inoculated into media and results were recorded after 21 days of incubation period. To obtain more exactly MIC and MBC, growth condition (agar dilution method) of $C$. albicans cultured on the media containing different concentrations of extract $(1,2,4,8,16,32,64,128$ and $256 \mathrm{mg} / \mathrm{ml})$ was studied. And in the last comparative study, for assessing the susceptibility of C. albicans, after detection of MBC and MIC of extract and amphotricine B (by micro broth dilution), the discs including $20 \mu \mathrm{g}$ of extract and amphotricin B $(100 \mathrm{U})$ have been made. MIC and MBC for 5\% extract were 3125 and 12500 (S. aureus ATTC 25923), 15670 and 6250 (S. epidermidis ATTC 12228) and 3125 and $6125 \mu \mathrm{g} / \mathrm{ml}$ (St. pyogones ATTC19615), respectively. The diameter of inhibition of zone for antibiotics varies from 30 to $56 \mathrm{~mm}$ and for $5 \%$ extract were $8-14 \mathrm{~mm}$. In the second study, yeast of $C$. albicans was grown in control tube but no growth was observed in media containing different $(35-250 \mathrm{mg} / \mathrm{ml})$ dilutions of extract. Trichophyton schenlaini and T. Verucosum were totally sensitive. Trichophyton rubrum and Microsporum gypsum were totally resistant. Trichophyton mentagrophyte, M. canis and Epidermophyton flucosum were resistant in 35,50 and $150 \mathrm{mg} / \mathrm{ml}$ dilution, but they were sensitive in dilution of $250 \mathrm{mg} / \mathrm{ml}$. Trichophyton violaseum was resistant in 35 and $50 \mathrm{mg} / \mathrm{ml}$ but sensitive in 150 and $250 \mathrm{mg} / \mathrm{ml}$. In the fourth study, growth of fungi was observed in all media except the media including concentration of extract of $1 \mathrm{mg} / \mathrm{ml}$ and in the fifth study, MBC and MIC of amphotricin B (amp B) was $(8,2 \mu \mathrm{g} / \mathrm{ml})$ and of extracts $5 \%(3125$ and $1569 \mu \mathrm{g} / \mathrm{ml})$. But this was 2 and $1 \mu \mathrm{g} / \mathrm{ml}$ for combination of extract and amp B. The inhibitional zone for amp B and $5 \%$ extract and both were 18,13 and $22 \mathrm{~mm}$, respectively. In the first study, $5 \%$ extract of plant has weakly anti-gram-positive bacterial effects. On the basis of the second study, this plant in mentioned dilutions has perfect anti-fungal effect against some common dermatopytes but not so good anti-fungal effect against $T$. rubrum and $T$. gypseum. The third study showed ethanolic extract of Echinophora platyloba has a perfect anti-C. albicans effect. Foruth study revealed a great promise in the application of extract of $E$. platyloba against $C$. albicans $(2 \mathrm{mg} / \mathrm{ml})$. In the fifth comparative study amp B has a potent anti C. albicans effect. MIC and MBC of $5 \%$ extract is 780 and 390 time less potent than amp B. But MIC and MBC of combination of both are two and four times more potent than amp B alone, which is a synergistic effect.

Poisonings from Herbs and Medicinal Plants Used for Self-medication in Italy: Epidemiology and Clinical Cases from Poison Control Centre of Milan

P. A. Moro ${ }^{1}$, F. Assisi ${ }^{1}$, M. Ferruzzi ${ }^{1}$, M. L. Colombo ${ }^{2}$, T. Della Puppa ${ }^{1}$ and F. Davanzo ${ }^{1}$

'Poison Control Centre, Niguarda Ca' Granda Hospital, Milan, Italy and

${ }^{2}$ Medicinal Plant Biology, Faculty of Pharmacy, University of Turin, Italy

The popular belief that all that is natural is healthy and harmless, widely promoted by mass media, induces people to use wild herbs as food, medicine and cosmetic. The species richness of endemic Italian flora and the easy availability of wild plants make the risk of picking up poisonous plants very high. People seem to be unaware that mistaking a poisonous plant for an edible one could be a very dangerous or a potential deadly occurrence. We reviewed all the calls concerning plant poisonings collected from PCC of Milan between 2001 and 2005 to point out the cases related to intentional use for healthy purpose. Among 4580 calls for suspected plant poisonings, 514 were closely related to intentional use of plants for self-treatment or health enhancement. The age range was 20-93 years. Aconitum ssp., Mandragora officinarum, Colchicum autumnale, Digitalis spp., were the more frequent and the more dangerous mistaken species: several life-threatening cases and three deaths were recorded. The misuse of Aloe, Lupinus, Ficus carica, and other species caused severe poisonings too. Some cases of poisonings were due to incorrect information diffused through radio, T.V., press and the Internet. Many species of poisonous plants and herbs grow wild in Italy. Severe or lethal poisonings may occur if similar species are mistaken or if medicinal plants are misused. A public information campaign on risks of picking up wild herbs and using them as food or as medicine would be promoted.We highlight the need for a better control on the content of messages diffused through mass media.

\section{Use of Herbal Products Among Pregnant Women in Empoli Sanitary} District

L. Gori, P. Martini, M. Lucchesi and F. Firenzuoli

Center of Natural Medicine, S. Giuseppe Hospital, Empoli, Italy

Use of unconventional therapies has almost doubled since 1991 among Italian people. Recent institutional surveys show that women use unconventional therapies more than men and not rarely during pregnancy too. Use of herbal products during pregnancy can be risky because there is very low experimental knowledge about fetal toxicity. The main objectives of the study was to spread and use of natural products in pregnant women and to inform and update obstetricians about natural products and uncoventional therapies. A survey design was used. The study was conduced in collaboration with the Gynecology 
and Midwifery operative unit of Empoli Hospital, Italy. From August to October 2005, we have interviewed 202 pregnant women with age between 35 and 44 years, during outpatient check-up and pre-natal course. A structured questionnaire was used to obtain detailed information regarding the use of herbal products during different pregnancy periods. Our population has been stratified by age: $15-25(5 \%), 26-35(73,9 \%)$ and $>36(21.1 \%)$. Common characteristics of the group: married with children, with a middle level of education and living in suburban area. Results show that the $20.3 \%$ (41 of 202) of the patients used natural products during pregnancy: herbal products $70.7 \%$; dietary supplements $26.8 \%$; vegetable drugs $17.1 \%$; homemade remedies $9.8 \%$; galenicals $0 \%$. Usage of herbal remedies during pregnancy: I trimester $46.3 \%$; II trimester $29.3 \%$; III trimester $34.1 \%$. No patient reported any relevant side effect. These women had many different sources of information: herbalist $51.5 \%$, television $39.1 \%$, friends and family $28.7 \%$, doctor $21.8 \%$, chemist $10.9 \%$, gynecologist $7.9 \%$, obstetrician $4 \%$, Internet $3 \%$ and other sources $2 \%$. Pregnant women chose herbal remedies as treatment of allergy $(96.5 \%)$, stress $(2 \%)$, gastrointestinal disease $(1.5 \%)$. Our study showed the results of a higher tendency to the use of herbal remedies during the first trimester of pregnancy, because of the higher risk of embriotoxicity. We think it is mandatory to inform and update the sanitary staff, and in particular midwives about the use of unconventional therapies by their patients, because of the growing use of these products and their potential toxical effects.

\section{Myopathy Associated with Herbal Medicine: Four Reports of Adverse Reactions to Fermented Red Rice}

Eugenia Gallo ${ }^{1}$, Alfredo Vannacci ${ }^{1}$, Francesca Menniti-Ippolito ${ }^{2}$, Luigi Gori ${ }^{3}$, Enrica Cecchi ${ }^{1}, 4$, Marina Di Pirro ${ }^{1}$, Grazia Banchelli ${ }^{1}$, Martina Moschini ${ }^{1}$, Francesco Lapi ${ }^{1}$, Roberto Raschetti ${ }^{2}$, Alessandro Mugelli ${ }^{1}$ and Fabio Firenzuoli ${ }^{3}$ ${ }^{1}$ Department of Preclinical and Clinical Pharmacology, University of Florence, 50139 Florence, ${ }^{2}$ Center of Epidemiology, Surveillance and Health Promotion, National Institute of Health, 00161 Rome, ${ }^{3}$ Centre of Natural Medicine, ASL 11 Empoli 50053 Empoli and ${ }^{4}$ Emergency Department, ASL 4 Prato Hospital, 59100 Prato, Italy

Herbal remedies (HRs) are often promoted to the public as possible alternatives to synthetic drugs. Moreover, the common perception that these products are 'natural' and therefore 'safe', could lead toward an inappropriate utilization. For example, myopathy, especially in case of rabdomyolysis, can be associated to HRs consumption. Among HRs, Ephedra (Ephedra sinica) and Commifora mukul have been described as causes of rabdomyolysis; different cases of acute myopathy can be linked to chronic or improper use of liquorice (Glycyrrhiza glabra) as well. Red yeast rice (Monascus ruber) extracts are widely merchandized as natural dietary cholesterol-lowering supplements. Monakolin $\mathrm{K}$, the main active principle, is identical to lovastatin, a well-known molecule able both to lower blood lipids and increasing the possibility of important side-effects on muscles, such as rabdomyolysis, even at very low doses.The aim of this study was to analyze the reports of myopathy associated to HRs, investigating the knowledge of healthcare professionals to identify this type of adverse reactions.Tuscan Regional Phytovigilance database includes 141 reports between 1 January 2001 and 31 May 2007. All spontaneous reports of myophathy were studied. Only four adverse reactions of myophaty, all associated with consumption of fermented red rice, were present in the database; two of them were sent by general practitioners, one by a specialist, one directly by a patient; three reactions were classified as 'probable', one 'certain'. No 'serious' reaction was reported. All cases showed an altered level of creatinine phosphokinase (CPK) serum level. Concerning the most 'serious' cases, one subject referred an increase of CPK $(401 \mathrm{UI} / \mathrm{ml})$, associated with muscle pain; the second one, who already accounted a positive history of adverse events to statins, developed a CPK increase $(357 \mathrm{Ul} / \mathrm{ml})$. All four cases recovered completely after discontinuation of treatment and, at 6 months of follow-up, good health conditions were confirmed. Among HRs, only fermented red rice was associated with muscle damage. All reports were classified at least as 'probable', the non-probable one was 'serious'. A causal association of myopathy with fermented red rice has been highlighted in the Tuscan regional database. These events were mild or moderate in severity. Furthermore, these data did not identify a relevant 'signal alarm' associated with red rice. Nevertheless, considering the level of under-reporting affecting every phytovigilance database, it cannot be excluded that some HRs could be responsible for 'serious' muscle disease. For this reason healthcare professionals should pay a major attention to serious AEs, not only associated to fermented red rice, but also other HRs.

The Nature and Experience of Adverse Effects Arising from the Delivery of Complementary and Alternative Medicine (CAM) to Children with Brain Injury-Initial Findings

A. Cheshire, L. Powell, J. Stapley and J. Barlow

Applied Research Centre in Health and Lifestyle Interventions:

Self-management Programme, Coventry University Priory Street

Coventry CV1 5FB, England

Research suggests that CAM use is prevalent among children with brain injury. As such, it is important that the risk of adverse effects is investigated. However, to date, there are few studies that have investigated this area. This study aims to investigate suspected adverse effects arising from the delivery of CAM to children with brain injury. Results presented here are initial findings which are part of an ongoing investigation. Parents of children with brain injury who used CAM for their child were identified through Cerebra, a charity for brain-injured children. A total of 39 parents completed a self-administered postal questionnaire regarding their child's CAM use and suspected adverse effects. Median number of CAM therapies used by children was 2 (range 1-5); the most popular therapies were osteopathy, massage, aromatherapy, reflexology and homeopathy. Nine (23\%) parents reported suspected adverse effects as a result of their child's CAM use. The number of adverse effects reported by each parent ranged between 1 and 3 , thus, a total of 15 adverse effects were reported altogether. Direct adverse effects included pain (4), bad temper, reaction to massage oils, withdraw symptoms when taken off certain foods, hyperactivity, severe diarrhea and behavior problems. Indirect adverse effects included distress at being held still for treatment (2), cost of treatment, travel time to treatment and missing school due to treatment. This study has demonstrated the importance of examining adverse effects in specific populations.

\section{Use of Herbal Remedies and Self-medication Among In-patients in} Empoli Hospitals

F. Firenzuoli, L. Gori and M. Lucchesi

Center of Natural Medicine, S. Giuseppe Hospital, Empoli, Italy

In Western countries and in Italy, a growing use of herbal drugs and selfmedication by herbal products and traditional preparations in the population has been observed. This survey has the aim to not only know the real uses and the modalities of preparation of herbs, but also and first of all to make a correct informative activity into the public sanitary structures by specific and educational programs: to collect systematically the information that concur to analyze and to comprise the supposition and the motivations of the crescent use of herbal drugs and self-medication by herbal preparations in the population; and to identify the possible risks for the health which derives by spontaneus harvesting. In the five afferent hospitals of the Asl 11 sanitary district of Empoli a cognitive surveying between the patients in several departments, on the use of herbal products has been carried out. The interview has been lead by specialized nonmedical staff through administration of a structured questionnaire. A total of 197 subjects (65.6\% women and $34.4 \%$ men) of medium age of 58 years have been interviewed. Of 197 interviews, it turns out that $48.7 \%$ patients use herbal remedies for health and cure of diseases like digestive, genito-urinary and cardiovascular disease; while $79.2 \%$ use herbals in the shape of infusions or decoctions for minor complaints or treatmant side-effects of synthetic drugs. The most important data is the high percentage of patients using herbal extracts $(20.8 \%)$. A Total of $35.4 \%$ followed the advice of an herborist, $11.4 \%$ of a pharmacist, $22.8 \%$ of a general practitioner and $20.3 \%$ practiced selfmedication. Around $14.4 \%$ cultivated herbs by themselves. They prepared infusions and decoctions and gave advices to other people too. Patients declared to get herbs in herbal shops in the $43.4 \%$ of cases, in pharmacy in $36.1 \%$, spontaneous harvesting in $14.4 \%$, supermarket in $6 \%$ and door to door in $1.2 \%$. A total of $20.8 \%$ of the interviewees used products made up of herbal products and traditional preparations. This percentage turns out to be much higher than official data of the Italian National Institute of Health $(4.8 \%)$ and of the Tuscany Region on phytotherapy $(5.6 \%)$. The disconcerting data is relative 
to the patients who carry out a self-medication by spontaneous harvesting in the fields (14.4\%). This survey also revealed that two patients used a plant that they thought sure because of the advise from popular practices. The plant was Teucrium chamaedrys L. known for its hepatotoxicity and formally forbidden by the Ministry of the Health because it is officially considered a poison as a simple decoction too.

Mixture of Feverfew and Yarrow Causes Beneficial Effects in Migraine Ilker Durak and Erdinç Devrim

Ankara University Faculty of Medicine Department of Biochemistry, 06100 - Ankara, Turkey

In folk medicine, it has been believed that both feverfew and yarrow exert therapeutic activities in patients with migraine. We aimed to investigate whethe mixture of feverfew and yarrow has indeed beneficial effects in patients with migraine headache. Mixture was prepared by using feverfew (chrysanthemum) and yarrow (Achillea millefolium) (2/1) as powder. Ten patients with migraine headache participated in the study. They consumed the mixture in a daily dose of 1 tea spoon $(\sim 2 \mathrm{~g})$ adding into 1 glass of light fatty milk for 6 months. It was observed that on a monthly base, the mean frequency of migraine attacks decreased from 3.5 to 1 after 1 month of use of the mixture and to almost none after 6 months. It was also observed that the severity of migraine attacks decreased considerably. It is suggested that consumption of mixture of feverfew and yarrow has beneficial and ameliorating effects in frequency and severity of migraine attacks in the patients. It has been suggested that it should be better for the patients with migraine headache to consume feverfew and yarrow as natural support products in their daily diet.

Garlic Oil Abolished Pancreatic Cyst in a Female Patient ilker Durak ${ }^{1}$, Hilmi Kocaoğlu ${ }^{2}$ and Erdinç Devrim ${ }^{1}$

${ }^{1}$ Department of Biochemistry and ${ }^{2}$ Department of Surgical Oncology, Ankara University Faculty of Medicine, Ankara 06100, Turkey

Since old ages, garlic has been used to treat several types of diseases. We aimed to investigate whether garlic oil exerts any beneficial effects in a patient with pancreatic cyst. A female patient of 43 years of age, diagnosed with pancreatic cyst participated in the study. She consumed garlic oil at a dose of $2 \mathrm{ml}$ a day for a month. Before and after the usage of garlic oil, the patient was examined by using ultrasound and magnetic resonance (MR). At the end of the study, it was observed by ultrasound and MR investigations that the pancreatic cyst was completely abolished and the patient ameliorated clinically. It is suggested that consumption of garlic oil exerts significant therapeutic effects in this case with pancreatic cyst.

\section{Mixture of Cinnamon and Fenugreek Powders Shows Beneficial Effects in Diabetic (type 2) Patients}

Erdinç Devrim and ilker Durak

Department of Biochemistry, Ankara University Faculty of Medicine, Ankara, 06100, Turkey

In folk medicine, it has been believed that both cinnamon and fenugreek exert blood glucose- regulating effects in diabetic patients. In the present study, we aimed to investigate whether the mixture of cinnamon and fenugreek powders have indeed regulatory effects in type 2 diabetic patients. Mixture was prepared by using Cinnamomum verum (cinnamon) and Trigonella foenumgraecum (fenugreek) $(2 / 1)$ as powder. Ten patients diagnosed with type 2 diabetes mellitus participated in the study. The patients consumed the mixture in a daily dose of 1 tea spoon $(\sim 2 \mathrm{~g})$ adding into 1 glass of light fatty milk for 2 months. They also continued to use their anti-diabetic drugs during the study. Before and after the study, blood samples were drawn from the patients to measure blood glucose levels. At the end of study, it was observed that blood glucose levels of the patients significantly decreased from $170 \pm 19$ to $130 \pm 14 \mathrm{mg} / \mathrm{dl} \quad(P<0.05)$. It is suggested that consumption of mixture of cinnamon and fenugreek has considerable effects in the regulation of blood glucose level in type 2 diabetic patients. It has been suggested that it should be better for the patients with type II diabetes mellitus to consume cinnamon and fenugreek as natural hypoglycemic products in daily diet in addition to their medical treatment.
Garlic Oil Exerts Beneficial Effects on Some Biochemical Parameters in a Patient with Parathyroid Cyst

Erdinç Devrim ${ }^{1}$, Hilmi Kocaoğlu ${ }^{2}$ and ilker Durak ${ }^{1}$

${ }^{1}$ Department of Biochemistry and ${ }^{2}$ Department of Surgical Oncology, Ankara University Faculty of Medicine, Ankara 06100, Turkey

Since old ages, garlic has been used to treat several types of diseases. We aimed to investigate whether garlic oil exerts any beneficial effects on some biochemical parameters in a patient with parathyroid cyst. A female patient of 38 years of age, diagnosed with parathyroid cyst participated in the study. Diagnosis was made by ultrasound examination and biochemical analyses. She consumed garlic oil at the dose of $2 \mathrm{ml}$ a day for a month. Before and after the usage of garlic oil, blood samples were drawn from the patient to measure parathyroid hormone (PTH), calcium $(\mathrm{Ca})$ and phosphorus $(\mathrm{P})$ levels in serum. At the end of the study, it was observed that serum PTH and Ca levels decreased from $112 \mathrm{pg} / \mathrm{ml}$ and $11.6 \mathrm{mg} / \mathrm{dl}$ to $16 \mathrm{pg} / \mathrm{ml}$ and $9.2 \mathrm{mg} / \mathrm{dl}$, respectively, and P level increased from $1.8 \mathrm{mg} / \mathrm{dl}$ to $3.2 \mathrm{mg} / \mathrm{dl}$. Additionally, it has been observed by ultrasound examination that cyst was completely eliminated after the use of the garlic oil. It is suggested that consumption of garlic oil exerts significant therapeutic effects in this case with parathyroid cyst.

Trypanocidal Component of the Volatile Fractions of Satureja macrantha S. Saeidnia ${ }^{1}$, A. R. Gohari ${ }^{1}$ and A. Hadjiakhoondi ${ }^{2}$

${ }^{1}$ Medicinal Plants Research Center and ${ }^{2}$ Department of Pharmacognosy, Faculty of Pharmacy, Medical Sciences/University of Tehran, Tehran, P. O. Box 14155-6451, Iran

Ether extract and the essential oil of Satureja macrantha, one of flavored species of Satureja belonging to Labiatae family, has shown the trypanocidal activity against the epimastigotes of Trypanosoma cruzi, the causative agent of American trypanomiasis. In this study, we aimed to isolate and identify the active component guided by in vitro bioassay. In order to fractionation, column chromatography and Lobar (Si-60) CC were used. All the fractions and separated compound were tested using in vitro anti-epimastigote activity test and then minimum lethal concentration (MLC) was determined. Many fractions from etheric extract and the essential oil, consisting of compound 1, have been observed active against epimastigotes of T. cruzi. Compound 1 was isolated and purified and then identified as thymol using ${ }^{1} \mathrm{H}$ and ${ }^{13} \mathrm{C}-\mathrm{NMR}$ spectra Thymol showed a good trypanocidal effect with $\mathrm{MLC}=25 \mu \mathrm{M}$ as compared to gentian violet as a positive control $(\mathrm{MLC}=6.2 \mu \mathrm{M})$. Volatile fractions of $S$. macrantha consists of thymol as an active volatile component against epimastigotes of $T$. cruzi, the ethiological agent of Chagas disease.

Immunosuppressive Principles from Achillea talagonica used in Iranian Traditional Medicine

S. Saeidnia ${ }^{1}$, N. Yassa $^{2}$, R. Rezaeipoor ${ }^{3}$, A. Shafiee $^{4}$, A. R. Gohari ${ }^{1}$ and M. Kamalinejad ${ }^{5}$

${ }^{1}$ Medicinal Plants Research Center, ${ }^{2}$ Department of Pharmacognosy, Faculty of Pharmacy, Medical Sciences/University of Tehran, ${ }^{3}$ Department of Immunology, School of Medicine, Shaheed Beheshti University of Medical Sciences,

${ }^{4}$ Department of Medicinal Chemistry, Faculty of Pharmacy and Pharmaceutical Research Center, Medical Sciences/University of Tehran and ${ }^{5}$ Department of Pharmacognosy, Faculty of Pharmacy, Shaheed Beheshti University of Medical Sciences, Tehran, P. O. Box 14155-6451, Iran

Achillea talagonica Boiss., belonging to Asteraceae family, grows in the western and central parts of Iran. This plant has long been used in traditional medicine for treatment of neuralgia and rheumatic pain. Previously, the immunosuppressive activity of the aqueous extract of this endemic plant was reported in experimental animals. In order to find the main immunosuppressive components of $A$. talagonica, $\mathrm{MeOH}$ and aqueous methanol $(80 \%$ and $50 \% \mathrm{v}: \mathrm{v})$ extracts were injected to BALB/c mice and the hemagglutinating antibody titer produced after SRBC (sheep red blood cells) immunization was assayed as a specific antigen. Guided by this assay, separation of active principles was done using column and paper chromatography methods. $\mathrm{ED}_{50}$ for pure compounds and prednisolone was determined by Probit analysis method using computer and SPSS statistical software. Isolated compounds were identified as caffeic acid 4-O-glucoside, quercetin, luteolin, 3'-methoxy luteolin, proline and choline by comparson of their spectral data with those reported in literatures. Immunosuppressive property of choline $\left(\mathrm{ED}_{50}: 5 \mathrm{mg} / \mathrm{kg}\right)$ was comparable to those of prednisolone $\left(E D_{50}: 10 \mathrm{mg} / \mathrm{kg}\right)$ although, quercetin $\left(E D_{50}: 30 \mathrm{mg} / \mathrm{kg}\right)$ and caffeoyl glucoside $\left(E_{50}: 20 \mathrm{mg} / \mathrm{kg}\right)$ decreased anti-SRBC titer compared with 
control groups. Immunosuppressive effect of $A$. talagonica is due to some components belonging to betaine, flavonol and phenoilc esters. Further study is required to find out a place for this plant and their active compounds in human therapies.

\section{Neural Therapy-Omeosiniatria Integrated to the Osteopathic Therapy,} in Patients with Post-traumatic Algodystrophy of the Right Arm Marco Loberti ${ }^{1}$ and Daniele Della Posta ${ }^{2}$

Viale A. Ballarin 700142 Roma and Via Fermo 2c 00182 Roma, Italy

A patient was involved in a car accident in June 2006, with a fracture of the third distal of the right humeri. He was hospitalized immediately and underwent surgical treatment for the apposition of the instrument of metallic synthesis of the rima of the fracture (Omeosiniatria); in the post-surgical phase, the patient was submitted to physical treatment for rehabilitation. Thereafter the patient followed serial controls of the injury through X-rays of the injured arm; 6 months later the fracture did not show any sign of bone reconstitution, the rima of the fracture was very similar to that of the initial control and moreover generated symptoms represented by: pain from the section proximal of the extended limb until the third distal of the forearm. Functional block of the elbow articulation both in flexion and in extension. Hypertonia of the muscles proximal of the limb and the skin over the injury and the zone of the seam appeared dystrophic, hypothermic and dyschromic compared with the surrounding skin. At follow-up, not having regained the functional recuperation of mobility and of the trophic state of the tissues, notwithstanding medical cure and physio-therapy in January 2007, the patient decided to visit an osteopath where he got a neurological evaluation. The articular range of motion was evaluated during the first visit and checked after 2-3 and 6 months highlighting a great gain (recovery) of the articular mobility. The muscular force of the brachial biceps(BB), triceps(TRI) and brachio radialis(BR) of the involved arm was evaluated using the MRC (medical research council) scale. At the beginning of the treatment the data recorded were 3 for the TRI were 3 and 4 for $B B$ and BR. At the end of the treatment the data were $4+$ for the $\mathrm{TRI}$ and 5 for $\mathrm{BB}$ and BR. Once the algodystrophic condition and distorted regulation were diagnosed, we decided to submit the patient to an integrated therapy according to our intervention model. The patient began a period of osteopathic care, every 2 weeks, with the technique of tissue normalization aimed at restoring the normal state of tension and of activity regulation of the extracellular matrix; this cure was integrated by treatment of infiltration with local anesthetics with therapeutic blocks of the omolateral stellate ganglium and of the seam. The name of this method is Neuraltherapy, a specific therapy for the regulation of the autonomic nervous system and consist in infiltrative technique with volumetric doses of 2cc of physiological sodium chloride solution mixed with $1 \%$ of local anesthetic - like lidocaine. This is a medical treatment and must be performed exclusively by a physician, better by a neurologist or anesthesiologist. Besides the neuraltherapy he was also given infiltration with homotoxicologic remedies in reflexpoints of the arm and of the tissues in next to the rima of the fracture. Additional homotoxicologic remedies were prescribed with intramuscle therapy. The integrated treatment lasted 5 months during which the motor conditions of the right arm improved progressively up to total recovery of the function of the elbow and of the shoulder, with an associated reduction of pain and of dystrophic disease of the tissue, the muscles and the bone. After 5 months of treatment, when additional X-rays of the fracture were taken, a slight reduction of the rima of the fracture was noticed. The therapeutic method used in the treatment of this clinical case was chosen to intervene in a condition of algodystrophy post-trauma. By definition, this pathology is characterized by a regulatory disturbance typically neurovegetative that in turn generates an incapacity of the healing process to overcome the various inflammation and reparation phases. To address this incapacity, we used therapeutic methods aimed at re-establishing a reparative physiological answer.

Representation of the Rachis in the Auricular Microsystem: a Puzzles Solution

Giancarlo G. Bazzoni

G.S.A.T.N. Gruppo di Studio sull'Agopuntura e le Terapie Naturali - F.I.S.A. Via Budapest, 807100 Sassar, Italy

Auricular therapy (also known as auricular acupuncture) was introduced in the fifties by the lyonese doctor P.Nogier and is well establiished world wide. In addition to the first studies produced by the French school regarding this approach, others were presented over the course of time, including most importantly the Chinese studies. Each of the aforementioned pieces of research developed its own auricular maps which differ greatly from one another. This work revolutionized existing scientific literature with regard to the description of the rachis in auricular acupuncture. The Chinese maps versus the French school approach will be the subject of careful analysis. In particular, the differing representation of the rachis and the integration of the ideas of two schools using a new clinical and neurophyisiological model will be explored (the aforementioned model is the fruit of exhaustive clinical practise by the author and his colleagues). The auricular description of the effect of the rachis on the 'antelix' was the first, in chronological order to be studied by Nogier. In the scope of the French school's research, the auricular map had been modified, but however, the exact location of the rachis level with the antelix remains the same. According to Nogier, the description of the rachis includes the antelix and it's lower root (crus antelicis inferior), the cervical column is located in the lower tract, the lumbar-sacral column in the final tract, the coccyx at the distal extremity, hidden from the ascending branch of the helix. Furthermore, R. Bourdiol and the Gemmer school believe that a detailed description of all the vertebral and paravertebral structures is possible. The invertebral discs, the interapofisari ligaments, the posterior and yellow vertebra, the meninx which bears on the antelical crest, a real barrier between the periferal area, where the muscles and paravertebral ligaments are located and the internal area which juts out into the vessel (containing the sympathetic nervous lateral-vertebral chain. Nogier, Bourdiol and Bahr retain that on the exterior of the media auricular (mastoidea) the description of the motor components of the rachis (which project onto the lower part of the anthelix and onto the rift of the inferior root of the anthelix can be found. The cervical tract is located in a lower position to that of the lumbar-sacral one on the ascending part. Bourdiol also agrees with this description which distinguishes between the two zones: the internal area containing the sympathetic nervous system of the ganglion chain whereas the external one contains the vertebral musculature. The first Chinese ear acupuncture was proposed in 1959 only 2 years after Nogier's original map drawn up by Bachmann. The author Xu Zuo Lin presents 15 new points in addition to the 45 existing points already proposed by the French school of thought. Over time the Chinese maps became even more individual moving away from those of Nogier and the French school. Although developed contemporarily in the seventies the Chinese maps differ significantly from the French ones regarding the localization of certain points. In the Chinese map, the description of the rachis bears upon the anthelix following the inverse somatotopic classification, the inferior cervical tract and the lumbar-sacral distal one whilst in the French map the rachidial projection extends right along the anthelix and the inferior root. The Chinese one is concerned only with the anthelix. The sacrococcyx tract in the Chinese example corresponds with the last dorsal vertebrae of the French one. The final dorsal tract of the Chinese map coincides with the initial part of the French one. Considering that auricular points and areas are the description of the innervation of an organ and a territory, the differing auricular descriptions of the rachid could be the result of a missing relationship between the 'scleromer' (the bone and tendon area innervated by a single pair of sensorial roots and their ganglion) and the 'dermatome' (the cutaneous area innervated by a single pair of sensorial roots and their ganglion). This could be explained by the anatomical 'covering' of the dorsal roots which moving distally tend to innervate even distant cutaneous areas. In the cutaneous map, the anthelix laid over allows us to see a somatotopic description very similar to that of the French maps. On the other hand, the rachidic anthelixal description of the Chinese school could be interpreted as the projection of different vertebral scleromer.

\section{Toxicity of Medicinal Herbs and Phytopharmacovigilance}

F. Borrelli, R. Capasso, G. Aviello, A. A Izzo and F. Capasso

Department of Experimental Pharmacology, University of Naples Federico II, Via D. Montesano 49, 80131 Naples, Italy

Medicinal herbs are enjoying a growing popularity in many industrialized countries. This trend has been promoted by several factors: (i) the idea that what is natural can only be good; (ii) the belief that herbs are completely innocuous in contrast to conventional drugs (licensed medicinal products); (iii) the presence of new diseases with severe complications for which there is still no satisfying treatment; (iv) the recognition of the value of the traditional medical system, particularly of Asian origin; and (v) the identification of plants that have been shown to have significant healing power. Problems of toxicity have recently surfaced for several herbs, challenging the safety reputation of phytotherapy. Considering this scenario, the aim of the present work was to identify all published case reports and case series regarding serious or potentially serious adverse effects of top-selling medicinal herbs. Four electronic literature databases were searched (Medline, Embase, Amed and Cochrane Library) all from their respective inception to May 2007. Bibliographies of the articles thus located 
were scanned for further relevant publications. Information was also sought from the spontaneous reporting programmes of the WHO. No language restrictions were imposed. The search terms used were: adverse drug reaction, adverse effect, adverse event, case report, safety, medicinal herb, medicinal plant, herba preparation and natural product. Numerous case reports and case series have been retrieved. However, identified data do not provide sufficient evidence that the herbal preparations are responsible for all the adverse reactions; many other causes or factors such as concomitant medications and the presence of contaminants/adulterants in the herbal preparation could contribute to the occurrence of the adverse events. The toxicity of herbal medicines is difficult to also demonstrate in consideration that a large number of herbs have not been submitted to rigorous quality control. In the interest of the consumer, we should develop, with a scientific rigor, the pharmacovigilance of herbal medicine (phytopharmacovigilance), i.e. the study of the safety of marketed herbal preparations and the practical condition of clinical usage in large communities.

\section{New Dietary Supplement from Raspberry (Rubus idaeus) \\ A. Crupi \\ Pharmalife Research, Lecco, Italy}

From red raspberry fruits (Rubus idaeus), we have today a major aromatic compound, the raspberry ketone [RK; 4-(4-hydroxyphenyl)butan-2-one] with an anti-obese action. The structure is similar to the structures of capsaicin and synephrine. Some recent scientific researches describe the pharmacologica actions of RK: RK enhances noradrenaline-induced lipolysis in isolated fat cells in vivo; the oral supplementation and topicall application of RK inhibits the fat subcutaneous accumulation, without any changes in the cardiovascular system; RK increases the energy consumption by stimulating metabolism.

The raspberry extract was evaluated for possible lypolitic effect in mice fed with high-fat diets. Forty-four-week-old male mice $(n=30)$ were fed either a normal diet (10 kcal fat $\%$ ) (10 mice) or high- fat diet (60 kcal fat\%) (20 mice) for 12 weeks. Feed consumption was not different among the groups, but energy intake was higher in the groups fed with high-fat diets than in the groups fed with normal diets. In the high-fat diet mice group there was higher body weight gain, abdominal fat accumulation and low-density lipoprotein (LDL)-cholestero in serum and higher triglyceride accumulation in liver. After 12 weeks, the highfat group was divided in two groups of 10 mice. The raspberry extract was administered to group 1 and normal high-fat diet to the other group. After 8 weeks, the raspberry group showed a $15 \%$ less weight than the verum group and $10 \%$ lower cholesterol and $7 \%$ lower triglycerides. At autopsy no mouse in the treated group showed any abnormal finding. Accordingly, the current results suggest that the oral administration of ASE seemed to lower the weight gain, serum LDL-cholesterol concentration and liver triglycerides accumulation in mice with obesity induced by high-fat diets.

Traditional Health Views and Cultural Mediation: a Way for Starting up Cultural Sensitivity

Bonciani Manila, Cangioli Benedetta and Confaloni Elisabetta

The debate on cultural mediation models, which are presently applied within health services, usually does not deal with the relationship between biomedicine, which is the reference paradigm of health services within nationa health systems and traditional health views of migrant groups. Actually cultural mediation, in the current use-seen as a system providing different services according to the different cultural backgrounds and health needs of usersincludes, among its many functions, the dialog between health professionals and migrants, also through the employment of cultural-linguistic mediators, where possible. Moreover it includes the training of health professionals in the perspective of 'starting up cultural sensitivity' (Declaration of Amsterdam, 2004).
With respect to these two functions, the comparison between traditional and biomedical etiology-as well as between health views, culture and health cultures-is already active, also where it is not explicit. In the first case, the people themselves call on their own cultures in their confontations and dialogs; in the second case, the 'starting up of cultural sensitivity' must pose a methodological question about 'how' and 'if' to deal with traditional medical and health views of migrants in order to inform the professionals about that, as mechanism for improving health professionals competences and implementing knowledge belonging to related contexts. It is particularly relevant for health promotion and migrant's health literacy improvement to wonder whether traditional knowledge of migrants are fully entitled to be included in health literacy, which is seen as all the necessary personal competences to take active charge of her/his own health. Indeed many prevention and self-cure actions come out from traditional knowledge. Some researches and experiences, such as the program Almaplanta of Almamater Centre in Turin, seem to substantiate this opinion, while models centered on mediator's neutrality seem to give methodological indications that it is not convenient nor necessary to deal with these knowledge. In Tuscany, the Albero della salute, during its experience inside health services, first in Prato and then in the whole region, experimented a model of integrated mediation, inspired by the ethno-psychiatric approach, which considers possible and useful to deal with traditional knowledge, both during the training for health professionals and in the relation with migrants' groups and communities, as well as in the actions addressed to implement health literacy like within the regional programe Mum Health carried out in 2006. This contribution means to discuss the theoretical and methodological questions aforementioned, and to describe the experiences of the Albero della salute in Tuscany.

Modulation of CD25 Dependant T cell Activation by Bromelain E. R. Secor Jr., A. Singh, L. A. Guernsey, C. M. Schramm and R. S. Thrall University of Connecticut School of Medicine, Department of Immunology MC-1319, 263 Farmington Avenue, Farmington, CT 06030-,1319, USA

Bromelain $(\mathrm{Br})$, an extract from the stems of common pineapple (family Bromeliaceae, order Bromeliales), is a natural product which is used to treat numerous inflammatory and immunological conditions, from arthritis and ulcerative colitis to cancer and allergy. $\mathrm{Br}$ is a cysteine protease and has been shown to both up-regulate and inhibit specific cell surface markers on distinct cell populations in a variety of immunological systems. Previous work in our laboratory has demonstrated that $\mathrm{Br}$ attenuates $\mathrm{CD} 4^{+}$- dependant airway inflammation via a reduction in eosinophils, $\mathrm{CD}^{+}$lymphocytes and inflammatory cytokines (IL-13) in an ovalbumin-induced allergic airway disease (OVA-AAD) model. This study was designed to determine the effect of in vitro $\mathrm{Br}$ treatment on $\mathrm{CD}^{+}$ splenic T cells through assessment of specific activation markers such as CD25 which is up-regulated on $T$ cells as they respond to antigen challenge and enhance the allergic response. CD4 ${ }^{+}$CD25 $5^{-}$Teff cells $\left(1 \times 10^{6}\right)$ were isolated (MACS ${ }^{\circledR}$ Cell Separation; Miltenyi Biotec) from the spleens of naïve mice and stimulated with plate bound $\alpha \mathrm{CD} 3 \mathrm{Ab}(10 \mathrm{mg} / \mathrm{ml}) . \mathrm{Br}(25,50$ or $100 \mathrm{mcg} / \mathrm{ml})$ was added to wells and cultures were harvested after $48 \mathrm{~h}$ and analyzed via flow cytometry for T-cell activation markers as compared to culture media controls. Antibodies used for flow cytometric analysis included: $\alpha$ CD4 (L3T4), $\alpha$ CD25 (PC61), $\alpha$ CD62L (MEL-14), aCD11a (M17/4) and $\alpha$ CD44 (IM7); (eBiosciences). Exogenous IL-2 (100 units; $\mathrm{NIH})$ and $\mathrm{E} 64$ (100 $\mathrm{mcg} / \mathrm{ml} ;$ Sigma-Aldrich) a cysteine protease inhibitor, were cultured separately with $\mathrm{Br}(100 \mathrm{mcg})$ to see if the reduction in CD25 could be rescued. In vitro $\mathrm{Br}$ treatment of $\alpha \mathrm{CD} 3$ stimulated $\mathrm{CD} 4^{+} \mathrm{CD} 25^{+} \mathrm{T}$ cells, reduced CD25, CD62L and CD44 as compared to media controls whereas CD11a was unaffected. Br treatment reduces CD25 in a dose-dependent manner which can be rescued by addition of exogenous IL-2 or E64 (data not shown). Br treatment of $\mathrm{CD}^{+} \mathrm{T}$ cells results in modulation of specific markers of activation and a selective reduction of CD25 in a dosedependent manner. These results may further our understanding of $\mathrm{Br}$ antiinflammatory and anti-allergy activity that has been described in both animal models and cell culture systems. 


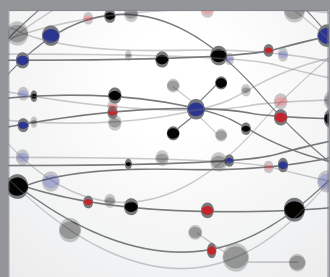

The Scientific World Journal
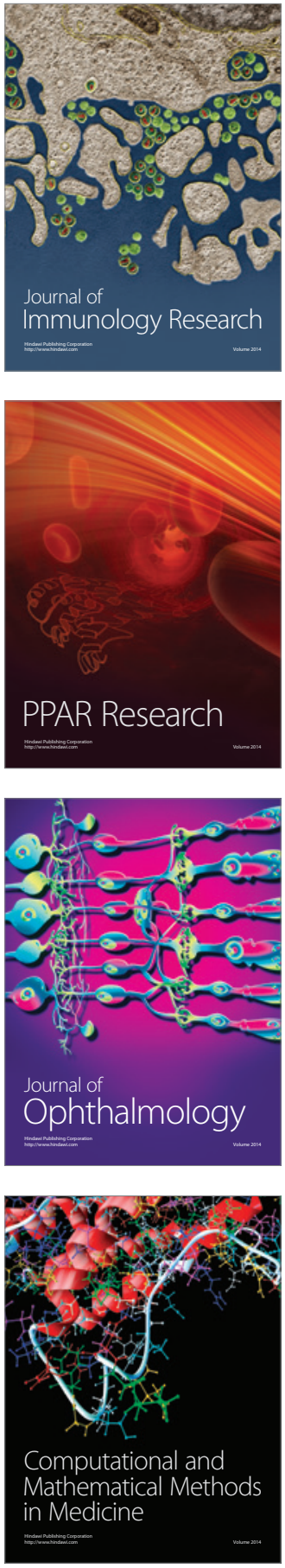

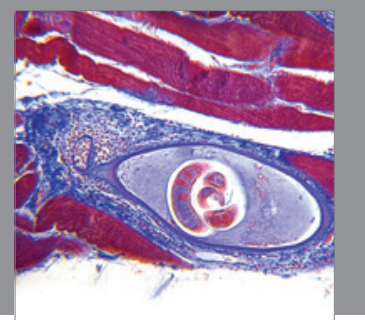

Gastroenterology

Research and Practice
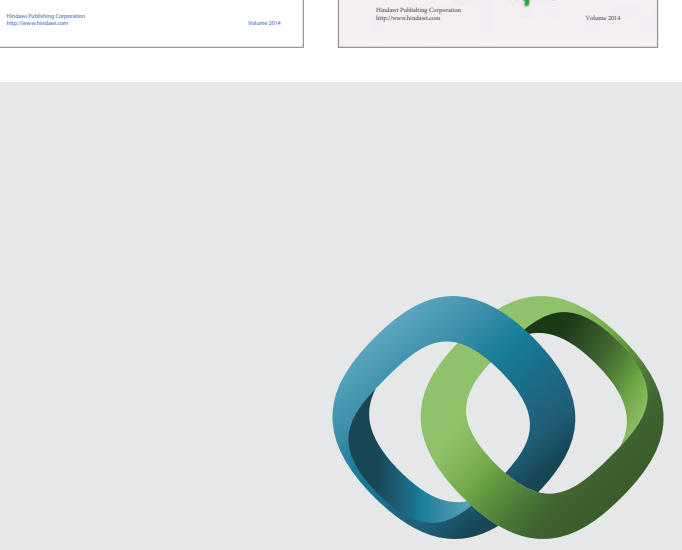

\section{Hindawi}

Submit your manuscripts at

http://www.hindawi.com
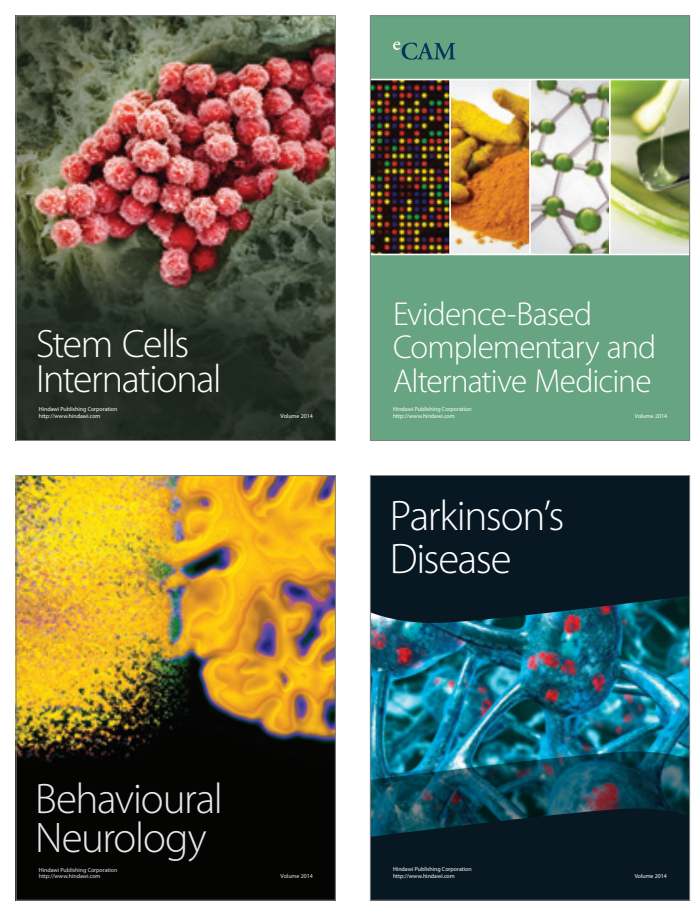

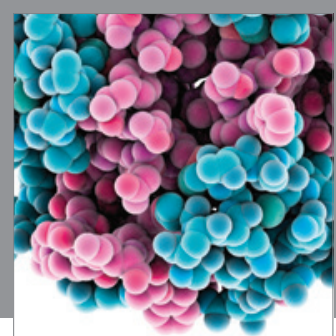

Journal of
Diabetes Research

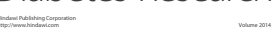

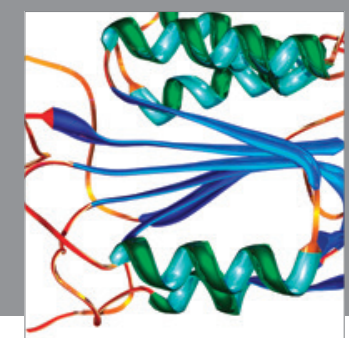

Disease Markers
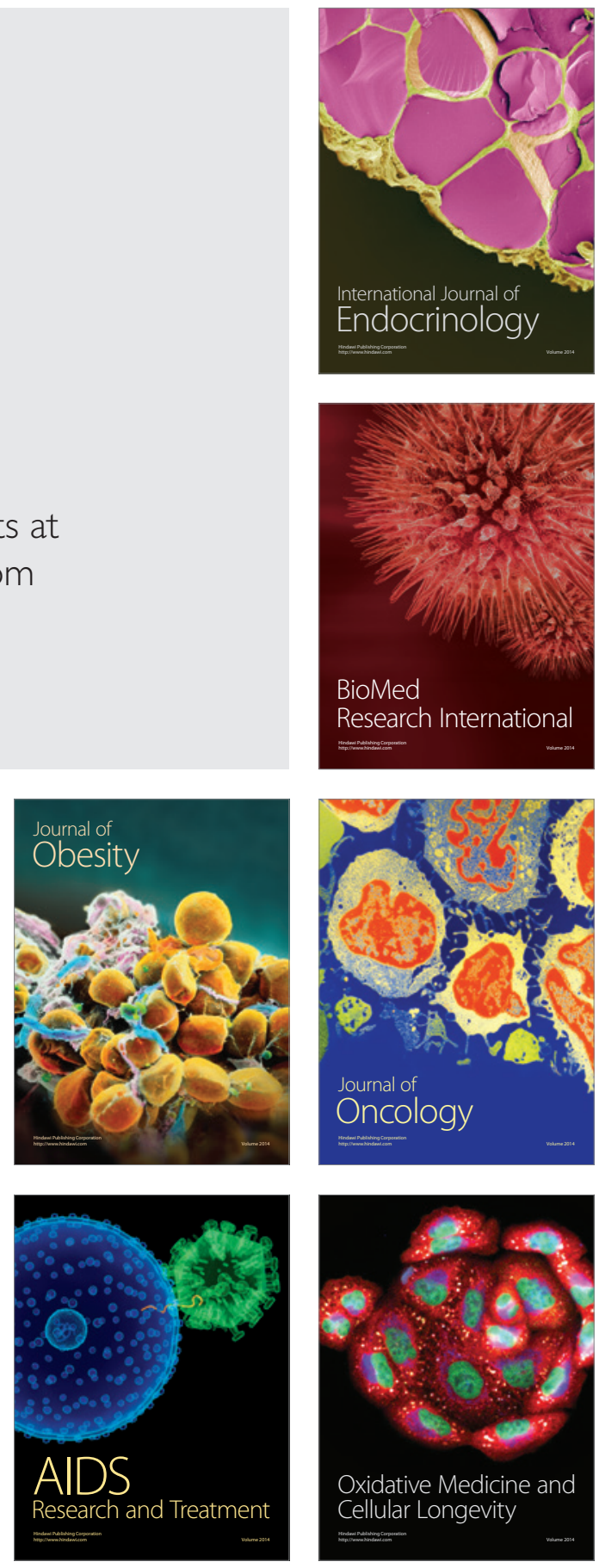